4.

\title{
ON CERTAIN EXPANSIONS, IN SERIES OF MULTIPLE SINES AND COSINES.
}

[From the Cambridge Mathematical Journal, vol. III. (1842), pp. 162-167.]

IN the following paper we shall suppose $\epsilon$ the base of the hyperbolic system of logarithms; $e$ a constant, such that its modulus, and also the modulus of $\frac{1}{e}\left\{1-\sqrt{1-e^{2}}\right\}$, are each of them less than unity; $\chi\left\{\epsilon^{u N(-1)}\right\}$ a function of $u$, which, as $u$ increases from 0 to $\pi$, passes continuously from the former of these values to the latter, without becoming a maximum in the interval, $f\left\{\epsilon^{u N(-1)}\right\}$ any function of $u$ which remains finite and continuous for values of $u$ included between the above limits. Hence, writing

$$
\chi\left\{\epsilon^{u N(-1)}\right\}=m
$$

and considering the quantity

$$
\frac{\sqrt{1-e^{2}} f\left\{\epsilon^{u N(-1)}\right\}}{\sqrt{-1} \epsilon^{u N(-1)} \chi^{\prime}\left\{\epsilon^{u N(-1)}\right\}(1-e \cos u)}
$$

as a function of $m$, for values of $m$ or $u$ included between the limits 0 and $\pi$, we have $\frac{\sqrt{1-e^{2}} f\left\{\epsilon^{u N(-1)}\right\}}{\sqrt{-1} \epsilon^{u N(-1)} \chi^{\prime}\left\{\epsilon^{u N(-1)}\right\}(1-e \cos u)}=\frac{2}{\pi} \Sigma_{-\infty}^{\infty} \cos r m \int_{0}^{\pi} \frac{\sqrt{1-e^{2}} f\left\{\epsilon^{u N(-1)}\right\} \cos r m d m}{\sqrt{-1} \epsilon^{u N(-1)} \chi^{\prime}\left\{\epsilon^{u N(-1)}\right\}(1-e \cos u)} \ldots(3)$,

(Poisson, Mec. tom. I. p. 650); which may also be written

$\frac{\sqrt{1-e^{2}} f\left\{\epsilon^{u N(-1)}\right\}}{\sqrt{-1} \epsilon^{u N(-1)} \chi^{\prime}\left\{\epsilon^{u N(-1)}\right\}(1-e \cos u)}=\frac{2}{\pi} \Sigma_{-\infty}^{\infty} \cos r m \int_{0}^{\pi} \frac{\sqrt{1-e^{2}} f\left\{\epsilon^{u N(-1)}\right\} \cos r \chi\left\{\epsilon^{u N(-1)}\right\} d u}{1-e \cos u} \ldots(4)$

and if the first side of the equation be generally expansible in a series of multiple cosines of $m$, instead of being so in particular cases only, its expanded value will always be the one given by the second side of the preceding equation. 
Now, between the limits 0 and $\pi$, the function

$$
f\left\{\epsilon^{u N(-1)}\right\} \cos r \chi\left\{\epsilon^{u N(-1)}\right\}
$$

will always be expansible in a series of multiple cosines of $u$; and if by any algebraical process the function $f \rho \cos r \chi \rho$ can be expanded in the form

$$
f \rho \cos r \chi \rho=\Sigma_{-\infty}^{\infty} \alpha_{8} \rho^{s}, \quad\left(\alpha_{8}=\alpha_{-\delta}\right)
$$

we have, in a convergent series,

$$
f\left\{\epsilon^{u N(-1)}\right\} \cos r \chi\left\{\epsilon^{u N(-1)}\right\}=\alpha_{0}+2 \Sigma_{1}^{\infty} \alpha_{8} \cos s u
$$

Again, putting

$$
\frac{1}{e}\left\{1-\sqrt{1-e^{2}}\right\}=\lambda
$$

we have $\cdot$

$$
\frac{\sqrt{1-e^{2}}}{1-e \cos u}=1+2 \Sigma_{1}^{\infty} \lambda^{p} \cos p u
$$

Multiplying these two series, and effecting the integration, we obtain

$$
\frac{1}{\pi} \int_{0}^{\pi} \frac{\sqrt{1-e^{2}} f\left\{\epsilon^{u N(-1)}\right\} \cos r \chi\left\{\epsilon^{u N(-1)}\right\} d u}{1-e \cos u}=2\left\{\frac{1}{2} \alpha_{0}+\Sigma_{1}^{\infty}\left(\alpha_{\delta} \lambda^{s}\right)\right\}
$$

and the second side of this equation being obviously derived from the expansion of $f \lambda \cos r \chi \lambda$ by rejecting negative powers of $\lambda$ and dividing by 2 , the term independent of $\lambda$ may conveniently be represented by the notation

$$
\overbrace{2 f \lambda \cos r \chi \lambda}
$$

where in general, if $\Gamma \lambda$ can be expanded in the form

$$
\Gamma \lambda=\Sigma_{-\infty}^{\infty}\left(A_{s} \lambda^{s}\right), \quad\left[A_{-s}=A_{s}\right]
$$

we have

$$
\overparen{\Gamma \lambda}=\frac{1}{2} A_{0}+\Sigma_{1}^{\infty} A_{8} \lambda^{s}
$$

(By what has preceded, the expansion of $\Gamma \lambda$ in the above form is always possible in a certain sense; however, in the remainder of the present paper, $\Gamma \lambda$ will always be of a form to satisfy the equation $\Gamma\left(\frac{1}{\lambda}\right)=\Gamma \lambda$, except in cases which will afterwards be considered, where the condition $A_{-8}=A_{8}$ is unnecessary.)

Hence, observing the equations (4), (9), (10),

$$
\frac{\sqrt{1-e^{2}} f\left\{\epsilon^{u N(-1)}\right\}}{\sqrt{-1} \epsilon^{u N(-1)} \chi^{\prime}\left\{\epsilon^{u N(-1)}\right\}(1-e \cos u)}=\Sigma_{-\infty}^{\infty} \cos r m \overbrace{2 \cos r \chi^{\lambda} f \lambda}
$$

from which, assuming a system of equations analogous to (1), and representing by $\Pi(\Phi)$ the product $\Phi_{1} \Phi_{2} \ldots$, it is easy to deduce

$$
\begin{aligned}
& \Pi\left\{\frac{\sqrt{1-e^{2}}}{\sqrt{-1} \epsilon^{u N(-1)} \chi^{\prime}\left\{\epsilon^{u N(-1)}\right\}(1-e \cos u)}\right\} f\left\{\epsilon^{u_{1} N(-1)}, \epsilon^{u_{2} N(-1)} \ldots\right\} \\
& =\Sigma_{-\infty}^{\infty} \Sigma_{-\infty}^{\infty} \ldots \Pi \cos r m \underbrace{\Pi(2 \cos r \chi \lambda) f\left(\lambda_{1}, \lambda_{2} \ldots\right)}
\end{aligned}
$$


where $\Gamma\left(\lambda_{1}, \lambda_{2} \ldots\right)$ being expansible in the form

$$
\begin{aligned}
\Gamma\left(\lambda_{1}, \lambda_{2} \ldots\right)= & \Sigma_{-\infty}^{\infty} \Sigma_{-\infty}^{\infty} \ldots A_{s_{1}, s_{2} \ldots} \lambda_{1}^{s_{1}} \lambda_{2}^{s_{2}} \ldots\left[A_{s_{1}}, s_{2} \ldots\right. \\
& \left.=A_{-s_{1}},-s_{2} \ldots\right] \\
& \Gamma\left(\lambda_{1}, \lambda_{2} \ldots\right)=\Sigma_{0}^{\infty} \Sigma_{0}^{\infty} \ldots \frac{1}{2^{N}} A_{s_{1}, s_{2} \ldots} \lambda_{1}^{s_{1}} \lambda_{2}^{s_{2}} \ldots, \ldots \ldots
\end{aligned}
$$

$N$ being the number of exponents which vanish.

The equations (13) and (14) may also be written in the forms

$$
\begin{aligned}
& f\left\{\varepsilon^{u N(-1)}\right\}=\Sigma_{-\infty}^{\infty} \cos r m \underbrace{2 \cos r \chi \lambda \frac{\sqrt{-1} \chi^{\prime} \lambda\left\{1-\frac{1}{2} e\left(\lambda+\lambda^{-1}\right)\right\}}{\sqrt{1-e^{2}}}} f \lambda \ldots \ldots \\
& = \Sigma _ { - \infty } ^ { \infty } \Sigma _ { - \infty } ^ { \infty } \ldots \Pi ( \operatorname { c o s } r m ) \longdiv { \Pi \{ 2 \operatorname { c o s } r \chi ^ { \lambda } \frac { \sqrt { - 1 } \chi ^ { \prime } \lambda \{ 1 - \frac { 1 } { 2 } e ( \lambda + \lambda ^ { - 1 } ) \} } { \sqrt { 1 - e ^ { 2 } } } \} f ( \lambda _ { 1 } , \lambda _ { 2 } \ldots ) } \ldots
\end{aligned}
$$

$f\left\{\epsilon^{u_{1} \Downarrow(-1)}, \epsilon^{u_{2} N(-1)} \ldots\right\}$

As examples of these formulæ, we may assume

$$
\chi\left\{\epsilon^{u N(-1)}\right\}=m=u-e \sin u
$$

Hence, putting

$$
\lambda^{r} \epsilon^{-\frac{r e}{2}(\lambda-\lambda-1)}+\lambda^{-r} \epsilon^{\frac{r e}{2}(\lambda-\lambda-1)}=\Lambda_{r}
$$

and observing the equation

$$
\sqrt{-1} \epsilon^{u N(-1)} \chi^{\prime}\left\{\epsilon^{u N(-1)}\right\}=1-e \cos u
$$

the equation (17) becomes

$$
f\left\{\epsilon^{u N(-1)}\right\}=\Sigma_{-\infty}^{\infty} \cos r m \Lambda_{r} \underbrace{\frac{\left\{1-\frac{1}{2} e\left(\lambda+\lambda^{-1}\right)\right\}^{2}}{\sqrt{ }\left(1-e^{2}\right)}} f \lambda
$$

Thus, if

$$
\theta-\varpi=\cos ^{-1} \frac{\cos u-e}{1-e \cos u}
$$

assuming

$$
f\left\{\epsilon^{u N(-1)}\right\}=\frac{\cos u-e}{1-e \cos u} .
$$

$$
\cos (\theta-\varpi)=\Sigma_{-\infty}^{\infty} \frac{1}{\sqrt{1-e^{2}}} \cos r m \overbrace{\left\{1-\frac{1}{2} e\left(\lambda+\lambda^{-1}\right)\right\}\left\{\frac{1}{2}\left(\lambda+\lambda^{-1}\right)-e\right\}} \Lambda_{r} \ldots
$$

the term corresponding to $r=0$ being

$$
\frac{1}{2 \sqrt{1-e^{2}}}\left\{2 \lambda-2 e-e\left(\lambda^{2}+1\right)+2 e^{2} \lambda\right\},=-e
$$

Again, assuming

$$
f\left\{\epsilon^{u N(-1)}\right\}=\frac{d \theta}{d m}=\frac{\sqrt{1-e^{2}}}{(1-e \cos u)^{2}}
$$


and integrating the resulting equation with respect to $m$,

$$
\theta-\varpi=\Sigma_{-\infty}^{\infty} \frac{\sin r m}{r} \underbrace{\Lambda_{r}}_{r}=m+2 \Sigma_{1}^{\infty} \frac{\sin r m}{r} \overbrace{\Lambda_{r}}
$$

a formula given in the fifth No. of the Mathematical Journal, and which suggested the present paper.

As another example, let

$$
f\left\{\epsilon^{u N(-1)}\right\}=\cos (\theta-\sigma) \frac{d \theta}{d m}=\frac{\sqrt{1-e^{2}}(\cos u-e)}{(1-e \cos u)^{3}}
$$

Then integrating with respect to $m$, there is a term

$$
2 m \frac{\overparen{\frac{1}{2}\left(\lambda+\lambda^{-1}\right)-e}}{\underbrace{1-\frac{1}{2} e\left(\lambda+\lambda^{-1}\right)}}
$$

which it is evident, a priori, must vanish. Equating it to zero, and reducing, we obtain

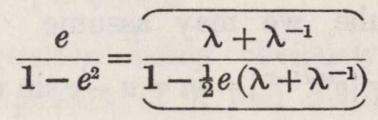

that is

$$
\frac{e}{1-e^{2}}=\lambda+\frac{e}{2}\left(\lambda^{2}+1\right)+\frac{e^{2}}{4}\left(\lambda^{3}+3 \lambda\right)+\frac{e^{3}}{8}\left(\lambda^{4}+4 \lambda^{2}+3\right)+\ldots
$$

a singular formula, which may be verified by substituting for $\lambda$ its value: we then obtain

$$
\sin (\theta-\varpi)=2 \Sigma_{1}^{\infty} \frac{\sin r m}{r} \underbrace{\frac{1}{1-\frac{1}{2}\left(\lambda+\lambda^{-1}\right)}}_{\Lambda_{r}^{\frac{1}{2}\left(\lambda+\lambda^{-1}\right)-e}}
$$

The expansions of $\sin k(\theta-\varpi), \cos k(\theta-\varpi)$, are in like manner given by the formulæ

$$
\begin{aligned}
& \cos k(\theta-\varpi)=\Sigma_{-\infty}^{\infty} \overbrace{\Lambda_{r} L^{\prime} \cos k L}^{\cos r m} \\
& \sin k(\theta-\varpi)=\Sigma_{-\infty}^{\infty} \overbrace{\Lambda_{r} \frac{1}{k r} \cos k L}^{\frac{\sin r m}{r}}
\end{aligned}
$$

where, to abbreviate, we have written

$$
\begin{gathered}
\cos ^{-1}\left\{\begin{array}{c}
\frac{1}{2}\left(\lambda+\lambda^{-1}\right)-e \\
1-\frac{1}{2} e\left(\lambda+\lambda^{-1}\right)
\end{array}\right\}=L \\
\frac{\left\{1-\frac{1}{2} e\left(\lambda+\lambda^{-1}\right)\right\}^{2}}{\sqrt{1-e^{2}}}=L^{\prime}
\end{gathered}
$$

Forming the analogous expressions for

$$
\cos k\left(\theta^{\prime}-\varpi^{\prime}\right), \quad \sin k\left(\theta^{\prime}-\varpi^{\prime}\right),
$$


substituting in

$\cos k\left(\theta-\theta^{\prime}\right)=\cos k\left(\varpi-\varpi^{\prime}\right)\left\{\cos k(\theta-\varpi) \cos k\left(\theta^{\prime}-\varpi^{\prime}\right)+\sin k(\theta-\varpi) \sin k\left(\theta^{\prime}-\varpi^{\prime}\right)\right\}$

$-\sin k\left(\varpi-\varpi^{\prime}\right)\left\{\sin k(\theta-\varpi) \cos k\left(\theta^{\prime}-\varpi^{\prime}\right)-\sin k\left(\theta^{\prime}-\varpi^{\prime}\right) \cos k(\theta-\varpi)\right\}$,

and reducing the whole to multiple cosines, the final result takes the very simple form

$\cos k\left(\theta-\theta^{\prime}\right)=\Sigma_{-\infty}^{\infty} \cos \left\{r^{\prime} m^{\prime}-r m+k\left(\varpi-\varpi^{\prime}\right)\right\} \Lambda_{r} \Lambda_{r^{\prime}}^{\prime} \cos k L \cos k L^{\prime}\left(L-\frac{1}{k r}\right)\left(L^{\prime}-\frac{1}{k r^{\prime}}\right)$

Again, formulæ analogous to (14), (18), may be deduced from the equation

$\Gamma\left(m_{1}, m_{2} \ldots\right)$

$$
=\Sigma_{-\infty}^{\infty} \Sigma_{-\infty}^{\infty} \ldots\left\{\begin{array}{r}
\cos \left(r_{1} m_{1}+r_{2} m_{2} \ldots\right) \int_{0}^{2 \pi} \frac{d m_{1}}{2 \pi} \int_{0}^{2 \pi} \frac{d m_{2}}{2 \pi} \ldots \cos \left(r_{1} m_{1}+r_{2} m_{2} \ldots\right) \Gamma\left(m_{1}, m_{2} \ldots\right) \\
+\sin \left(r_{1} m_{1}+r_{2} m_{2} \ldots\right) \int_{0}^{2 \pi} \frac{d m_{1}}{2 \pi} \int_{0}^{2 \pi} \frac{d m_{2}}{2 \pi} \ldots \sin \left(r_{1} m_{1}+r_{2} m_{2} \ldots\right) \Gamma\left(m_{1}, m_{2} \ldots\right)
\end{array}\right.
$$

which holds from $m_{1}=0$ to $m_{1}=2 \pi$, \&c., but in many cases universally. In this case, writing for $\Gamma\left(m_{1}, m_{2} \ldots\right)$ the function

$$
\Pi\left\{\frac{1}{\sqrt{-1} \epsilon^{u N(-1)} \chi^{\prime}\left\{\epsilon^{u N(-1)}\right\}} \frac{\sqrt{1-e^{2}}-e \sin u \sqrt{-1}}{1-e \cos u}\right\} f\left\{\epsilon^{u_{1} \downarrow}(-1), \epsilon^{u_{2} N(-1)} \ldots\right\} \ldots(40) ;
$$

and observing

$$
\frac{\sqrt{1-e^{2}}-e \sin u \sqrt{-1}}{1-e \cos u}=\frac{1+\lambda \epsilon^{-u N(-1)}}{1-\lambda \epsilon^{-u N(-1)}}=1+2 \Sigma_{1}^{\infty}\{\cos s u-\sqrt{-1} \sin s u\} \lambda^{s}
$$

an exactly similar analysis, (except that in the expansion

$$
\Gamma\left(\lambda_{1}, \lambda_{2} \ldots\right)=\Sigma_{-\infty}^{\infty} \Sigma_{-\infty}^{\infty} \ldots A_{s_{1}, s_{2}} \ldots \lambda_{1}^{s_{1}} \lambda_{2}^{s_{2}} \ldots,
$$

the supposition is not made that $\left.A_{s_{1}, s_{2}} \ldots=A_{-s_{1},-s_{2}} \ldots\right)$, leads to the result

$$
\begin{aligned}
& f\left\{\epsilon^{u_{1} N(-1)}, \epsilon^{u_{2} N(-1)} \ldots\right\} \Pi\left\{\frac{\sqrt{1-e^{2}}-e \sin u \sqrt{-1}}{\sqrt{-1} \epsilon^{u N(-1)} \chi^{\prime}\left\{\epsilon^{u N(-1)}\right\}(1-e \cos u)}\right\} \\
& =\Sigma_{-\infty}^{\infty} \Sigma_{-\infty}^{\infty} \cdots\left\{\begin{array}{r}
\cos \left(r_{1} m_{1}+r_{2} m_{2} \ldots\right) \underbrace{2^{2^{n} \cos \left(r_{1} \chi_{1} \lambda_{1}+r_{2} \chi_{2} \lambda_{2} \ldots\right) f\left(\lambda_{1}, \lambda_{2} \ldots\right)}} \\
+\sin \left(r_{1} m_{1}+r_{2} m_{2} \ldots\right) \overbrace{}^{2^{n} \sin \left(r_{1} \chi_{1} \lambda_{1}+r_{2} \chi_{2} \lambda_{2} \ldots\right) f\left(\lambda_{1}, \lambda_{2} \ldots\right)} \ldots
\end{array}\right.
\end{aligned}
$$

(n) being the number of variables $u_{1}, u_{2} \ldots$. Hence also $f\left\{\epsilon^{u_{1} N(-1)}, \epsilon^{u_{2} N(-1)} \ldots\right\}$

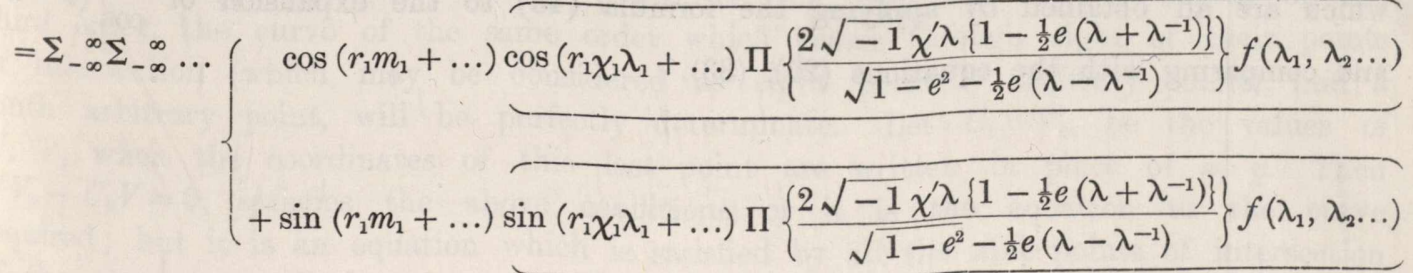


By choosing for $f\left\{\epsilon^{u_{1} N(-1)}, \epsilon^{u_{2} N(-1)} \ldots\right\}$, functions expansible without sines, or without cosines, a variety of formulæ may be obtained: we may instance

$$
\frac{\overparen{\left(\lambda-\lambda^{-1}\right)\left\{1-\frac{1}{2} e\left(\lambda+\lambda^{-1}\right)\right\} \Lambda_{r}}}{\sqrt{1-e^{2}-\frac{1}{2} e\left(\lambda-\lambda^{-1}\right)}}=0
$$

$\Lambda_{r}$ having the same meaning as before.

Also,

$$
\underbrace{=0}_{\frac{\left\{\frac{1}{2}\left(\lambda+\lambda^{-1}\right)-e\right\}\left\{1-\frac{1}{2} e\left(\lambda+\lambda^{-1}\right)\right\} \Lambda_{r}^{\prime}}{\sqrt{1-e^{2}}-\frac{1}{2} e\left(\lambda-\lambda^{-1}\right)}}=0
$$

where

$$
\Lambda_{r}^{\prime}=\lambda^{r} \epsilon^{-\frac{r e}{2}(\lambda-\lambda-1)}-\lambda^{-r} \epsilon^{\frac{r e}{2}\left(\lambda-\lambda^{-1}\right)}
$$

Again,

$$
\underbrace{\underbrace{\frac{1}{\Lambda_{r}}}}_{\frac{\left.1-\frac{1}{2} e\left(\lambda+\lambda^{-1}\right)\right\}\left(\lambda-\lambda^{-1}\right) \Lambda_{r}^{\prime}}{1-\frac{1}{2} \frac{e}{\sqrt{1-e^{2}}}\left(\lambda-\lambda^{-1}\right)}}=0
$$

and

$$
\underbrace{\frac{\left\{1-\frac{1}{2} e\left(\lambda+\lambda^{-1}\right)\right\}\left\{\left(\lambda+\lambda^{-1}\right)-\frac{1}{2} e\right\} \Lambda_{r}}{1-\frac{1}{2} \frac{e}{\sqrt{1-e^{2}}}\left(\lambda-\lambda^{-1}\right)}}=\underbrace{\left\{1-\frac{1}{2} e\left(\lambda+\lambda^{-1}\right)\right\}\left\{\left(\lambda+\lambda^{-1}\right)-\frac{1}{2} e\right\} \Lambda_{r}}, \ldots
$$

or, what is the same thing,

$$
\underbrace{\frac{\left(\lambda-\lambda^{-1}\right)\left\{1-\frac{1}{2} e\left(\lambda+\lambda^{-1}\right)\right\}\left\{\left(\lambda+\lambda^{-1}\right)-\frac{1}{2} e\right\} \Lambda_{r}}{1-\frac{1}{2} \frac{e}{\sqrt{1-e^{2}}}\left(\lambda-\lambda^{-1}\right)}}=0
$$

or, comparing with (44),

$$
\frac{\overparen{\left(\lambda^{2}-\lambda^{-2}\right)\left\{1-\frac{1}{2} e\left(\lambda+\lambda^{-1}\right)\right\} \Lambda_{r}}}{1-\frac{1}{2} \frac{e}{\sqrt{1-e^{2}}}\left(\lambda-\lambda^{-1}\right)}=0
$$

which are all obtained by applying the formula (43) to the expansion of $\frac{\sin }{\cos }(\theta-\varpi)$, and comparing with the equations (25), (33). 